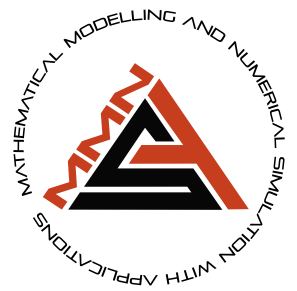

\title{
Second-grade fluid with Newtonian heating under Caputo fractional derivative: analytical investigations via Laplace transforms
}

\author{
Ndolane Sene $1, *, \neq$ \\ ${ }^{1}$ Department of Mathematics, Cheikh Anta Diop University, BP 5683 Dakar Fann, Senegal \\ * Corresponding Author \\ ‡ndolanesene@yahoo.fr (Ndolane Sene)
}

\begin{abstract}
In this paper, we consider the constructive equations of the fractional second-grade fluid. The considered fluid model is described by the Caputo derivative. The problem consists to determine the exact analytical solution using the Laplace transform method. The influence of the order of the used fractional operator has been presented in this paper. We also analyze the influence of the Prandtl number in the dynamics of the temperature distribution according to the variation of the order of the Caputo derivative. The impact of the second-grade parameter and the Grashof number in the dynamics of the velocity has been presented and discussed. The influences of the parameters used in the modeling have been interpreted in terms of a fractional context. In general, it is shown that the order of the fractional operator influences the diffusivity of the considered fluid. This influence can cause an increase or decrease in the temperature and velocity distributions. The main findings of the paper have been illustrated using the graphical representations of the considered distributions according to the order of the fractional operator.
\end{abstract}

Key words: Second-grade fluid; Grashof number; Prandtl number; Laplace transforms

AMS 2020 Classification: 26A33; 35C15; 42A38; 35K57

\section{Introduction}

The field of fractional calculus and its application has grown many attractions these last decade. There exist nowadays many theories and applications related to the field of fractional calculus [1]. The attraction of this new field is due to the memory effect and the heredity noticed in the fractional operators. We have many fractional operators as the Caputo derivative and the Riemann-Liouville derivative which are known as the fractional derivative with singular kernels $[2,3]$. We have also the so-called Atangana-Baleanu fractional operator and the Caputo-Fabrizio derivative which are known as the fractional operators with non-singular kernels $[4,5]$. These singular and non-singular derivatives appear in many papers with applications to physical modeling [2, 6, 7], biological modeling [8, 9, 10, 11, 12, 13, 14], sciences and engineering modeling [15, 16, 17, 18, 19], mathematical physics modeling [20, 21, 22, 23, 24, 25, 26], physics modeling [24, 27] and others domains $[28,29,30,31,32]$. The field of fractional calculus is interesting but there also exist many questions without responses. The following questions are asked in the field of fractional calculus: what is a fractional operator, why fractional operators, what are the advantages, and the motivations of using the fractional operators? Some of these questions have responses but some of them are still without concrete responses. Modeling fluid, and nanofluid with fractional operators have attracted many authors these last decade. The investigations related to modeling fluid and nanofluid with the fractional operators can be found in the following papers [20,33, 34]. The literature concerning the fluid and second-grade fluid models with fractional operators is long. In this part, we recall the literature review. In [33], the authors have proposed a model related to free convection flow near a vertical plate described by Caputo derivative and have considered its solution via Laplace transform method. In [20], the authors have taken into account the analytical solutions via Laplace

> Received: 13.12.2021 > Revised: 16.01.2022 > Accepted: 22.01.2022 > Published: 26.01.2022 
transform for a fractional double convection problem of fractional viscous fluid particularly described by a Caputo fractional operator. Ali et al. in [35] have proposed the exact analytical solution of MHD free convection flow of generalized Walters'-B fluid model described by new fractional operator namely Caputo-Fabrizio derivative. In [36], the authors have obtained a solution for the free convection flow of generalized Jeffrey fluid described by the Caputo-Fabrizio fractional. In [15], the authors have used the Laplace transform to get the exact analytical solution of the MHD flow of water-based Brinkman type nanofluid. In [37], the authors have used the Caputo derivative to model heat and mass transport of differential type fluid and have examined the exact analytical solution using the Laplace transform method. In [38], the authors have studied the unsteady MHD free convection flow of Casson fluid past over an oscillating vertical plate embedded in a porous medium, the Laplace transform has been used in such paper to get the analytical solutions. In [21], the authors have proposed a comparative study between the Caputo-Fabrizio derivative and Atangana-Baleanu derivative in modeling the generalized Casson fluid model with heat generation and chemical reaction. In [28], Tahir et al. have proposed the analytical solution of the heat transfer flow of Maxwell fluid described by Caputo-Fabrizio time-fractional derivative. In [39], the authors have studied the MHD flow of a Casson fluid over an exponentially shrinking sheet, the analytical solution of the proposed model has been proposed via the Adomian Decomposition Method. In the same direction of investigations related to the determination of the analytical solution using Laplace transform for the models with integer-order derivative see in [40].

In this work, we focus on the analytical solutions of the constructive equations of the second-grade fluid model described by the Caputo fractional operator. We use in this paper the Laplace transform method for getting the analytical solution. The advantages of the present investigations are we use the resolution of second-order differential equations which are not difficult to be performed. The second advantage of the present paper is the analytical solutions can be rewritten using the exponential function, the Mittag-Leffler function, the wright functions, and the Gaussian error function. The memories effect present in the Caputo derivative will also be an advantage in the present paper because the order of the Caputo derivative will play accelerations or retardation effect on the dynamics of the velocity and the temperature distribution of the considered model.

The contents of the present paper are structured as follows. In Section 2, we try to recall the fractional operators most used in the literature of fractional calculus. It will permit the readers to be familiarized with the fractional operator. In Section 3, we describe the fractional model using the Caputo derivative. In Section 4, we give the approaches to get the analytical solutions using the Laplace transform method. Note that the Laplace transform of the Caputo derivative will be frequently used. Discussion and the interpretations of the influences of the parameters utilized in the modeling have been provided in Section 5 . We finish the paper with final remarks in Section 6.

\section{Fractional operators}

This section is devoted to giving the definitions of the fractional operators and the functions which will be used in this investigation. For present works, we need the Caputo fractional operator, the Riemman-Liouville integral, the derivatives with son singular kernels, the Mittag-Leffler functions, the wright function, and others. We also will recall the Laplace transform of the Caputo derivative because this tool is fundamental in our investigations regarding the method utilized in the present paper. The Riemann-Liouville integral is described in the following definition.

Definition $1[2,3]$ The representation of the Riemann-Liouville integral of a considered function $g:[0,+\infty[\rightarrow \mathbb{R}$ can be expressed as the following form

$$
\left(I^{\alpha} g\right)(t)=\frac{1}{\Gamma(\alpha)} \int_{0}^{t}(t-s)^{\alpha-1} g(s) d s
$$

the $\Gamma(. .$.$) denotes the Gamma function and with order \alpha$ verifying the condition that $\alpha>0$.

The Riemann-Liouville integral has its associated fractional derivative known as the Riemann-Liouville derivative. We give its definition in the following definition. This definition can be found in many papers in the literature.

Definition $2[2,3]$ The representation of the Riemann-Liouville derivative of the considered function $g:[0,+\infty[\rightarrow \mathbb{R}$, of order $\alpha$ as the form

$$
D^{\alpha} g(t)=\frac{1}{\Gamma(1-\alpha)} \frac{d}{d t} \int_{0}^{t} g(s)(t-s)^{-\alpha} d s,
$$

the time $t>0$, is the order of the operator and satisfies the condition that $\alpha \in(0,1)$ and $\Gamma(.$.$) represents the Gamma Euler function.$

The Riemann-Liouville derivative has an increasing reputation in the problems related to the existence and the uniqueness, the stability analysis of the fractional differential problems. In modeling real words problems the initial condition makes this operator very limited because the real-world problems' initial conditions are not compatible with the Riemann-Liouville derivative. Therefore this derivative is not used in modeling biological models, fractional chaotic systems, and other real applications. The Caputo derivative is adequate in modeling real word problems and is defined in the following definition.

Definition $3[2,3]$ We denote the Caputo fractional derivative with the considered function $g:[0,+\infty[\rightarrow \mathbb{R}$, of order $\alpha$ as the following representation

$$
D^{\alpha} g(t)=\frac{1}{\Gamma(1-\alpha)} \int_{0}^{t} \frac{d g}{d s}(t-s)^{-\alpha} d s
$$

with $t>0$, and the order of the derivative obeys to the assumption that $\alpha \in(0,1)$ and $\Gamma(. .$.$) is the Gamma Euler function.$

The Caputo derivative is the most used derivative in the literature of fractional calculus. The motivation is due to the fact that this derivative is compatible with the initial conditions used in modeling real word problems. In this paper the Laplace transform method is used for getting the exact analytical solutions, therefore we define in the following line the Laplace transform of the Caputo derivative. We have the 
following representation $[2,3]$

$$
\mathcal{L}\left\{\left(D_{c}^{\alpha} g\right)(t)\right\}=s^{\alpha} \mathcal{L}\{g(t)\}-s^{\alpha-1} g(0) .
$$

with the order $\alpha$ satisfies the condition that $\alpha \in(0,1)$. The symbol $\mathcal{L}$ denotes the Laplace transform. The Laplace transform in Eq. (4) will play a fundamental role in the present investigation.

Before closing this section, we recall the definitions of the fractional operators with non-singular kernels. These derivatives have many applications these decades and many papers have been written to illustrate the applications of these derivatives in modeling physics, biology models, science, and engineering.

Definition 4 [5] We represent by the following equation of the Caputo-Fabrizio derivative of the function $g:[0,+\infty[-\longrightarrow \mathbb{R}$, of order $\alpha$ in the following term

$$
D^{\alpha, C F} g(t)=\frac{C F(\alpha)}{1-\alpha} \int_{0}^{t} g^{\prime}(s) \exp \left(-\frac{\alpha}{1-\alpha}(t-s)\right) d s,
$$

where the following the condition $t>0$, the order of the fractional derivative obeys to $\alpha \in(0,1)$ and $C F(\ldots)$ denotes the normalization term and respects to the condition $C F(0)=C F(1)=1$.

Definition 5 [4] The definition of the Atangana-Baleanu derivative of the function $g:[0,+\infty[\longrightarrow \mathbb{R}$, of order $\alpha$, that is

$$
D^{\alpha, A B} g(t)=\frac{A B(\alpha)}{1-\alpha} \int_{0}^{t} g^{\prime}(s) E_{\alpha}\left(-\frac{\alpha}{1-\alpha}(t-s)^{\alpha}\right) d s,
$$

respecting the condition that $t>0$, the order of the fractional derivative $\alpha \in(0,1)$ and $A B(\ldots)$ is the normalization term and obeys to the condition $A B(0)=A B(1)=1$.

We finish this section by recalling the special functions which are used to express the analytical exact solutions in this paper. We have the Mittag-Leffler function and the wright function represented in the following expressions [33],

$$
E_{\alpha, \beta}(x)=\sum_{k=0}^{\infty} \frac{x^{k}}{\Gamma(\alpha k+\beta)},
$$

with $\alpha>0, \beta \in \mathbb{R}$ and $x \in \mathbb{C}$, and we define the Wright function [33] with three parameters as the following

$$
\phi(\beta,-\sigma, x)=\sum_{n=0}^{\infty} \frac{x^{n}}{\Gamma(n+1) \Gamma(\beta-\sigma n)},
$$

with the following conditions $\sigma \in(0,1), \beta \in \mathbb{R}$ and $x \in \mathbb{C}$.

\section{Fractional model under Caputo derivative}

This section is devoted to the presentation of the second-grade fluid model subject of our investigations. To arrive at our end, we describe the following procedure. The sketch of modeling can be found in the literature in the following papers [20,33], the significant difference in the model is the initial condition which play important role in the form of the analytical solutions. We take the plate vertical at $x$-direction and we take $y$-direction perpendicular to the plane generated by the plane. We consider that at the initial time, then the fluid and the plate are at rest to the constant temperature $T_{\infty}$. At starting time, we suppose that the heat transfer from the plate to the considered fluid is proportional to a local surface temperature denoted by T. For the rest of our modeling, we consider the use of the Boussinesq approximation and then we get the following partial differential equations

$$
\begin{aligned}
\frac{\partial u}{\partial t} & =\left(v+\frac{\alpha_{1}}{\rho} \frac{\partial}{\partial t}\right) \frac{\partial^{2} u}{\partial y^{2}}+g \beta\left(T-T_{\infty}\right), \\
\frac{\partial T}{\partial t} & =\frac{\kappa}{\rho c_{p}} \frac{\partial^{2} T}{\partial y^{2}} .
\end{aligned}
$$

The initial and boundary conditions adopted in this present investigations are described as follows

$$
\begin{aligned}
& v(u, 0)=0, \quad T(x, 0)=T_{\infty}, \\
& T(0, t)=0, \quad T(0, t)=T_{\infty}+\left[T_{w}-T_{\infty}\right]\left[\frac{t}{t_{0}}\right] .
\end{aligned}
$$

The problem consists to get the exact analytical solutions of the model (9)-(10), and then we need more simplifications of the previous model. Therefore we introduce the following changes variables

$$
\begin{array}{r}
y^{*}=\frac{y h}{k}, \quad t^{*}=\frac{t}{\frac{1}{v\left(\frac{k}{h}\right)^{2}},} \quad u^{*}=\frac{u}{\frac{g}{v\left(\frac{k}{h}\right)^{2}}}, \quad \psi^{*}=\frac{T-T_{\infty}}{T_{\infty}}, \\
G r=\beta T_{\infty}, \quad \operatorname{Pr}=\frac{\rho c_{p}}{\kappa}, \quad \beta^{*}=\frac{\alpha_{1}}{\rho}\left(\frac{h}{k}\right)^{2} .
\end{array}
$$


We use the dimensionless variable described in Eq. (13) and Eq. (14) into Eq.(9) to Eq. (12), we get a more simplified form of the fluid model considered in this paper, it is represented by the following

$$
\begin{aligned}
\frac{\partial u}{\partial t} & =\frac{\partial^{2} u}{\partial y^{2}}+\beta \frac{\partial^{3} u}{\partial t \partial y^{2}}+G r \psi \\
\frac{\partial \psi}{\partial t} & =\frac{1}{\operatorname{Pr}} \frac{\partial^{2} \psi}{\partial y^{2}} .
\end{aligned}
$$

with initial and boundaries dimensionless conditions given

$$
\begin{aligned}
u(y, 0) & =\psi(y, 0)=0, \\
u(0, t) & =0, \\
\psi(0, t) & =t .
\end{aligned}
$$

Replacing the integer-order derivative by the Caputo derivative in Eqs. (15)-(16) due to the memory effect and the heredity of the Caputo derivative and the generalization of the integer-order derivative to non-integer partial differential equations, we get the following model which will be the subject of our investigations

$$
\begin{aligned}
D_{\tau}^{\alpha} u & =\frac{\partial^{2} u}{\partial y^{2}}+\beta D^{\alpha}\left(\frac{\partial^{2} u}{\partial y^{2}}\right)+G r \psi \\
D_{\tau}^{\alpha} \psi & =\frac{1}{\operatorname{Pr}} \frac{\partial^{2} \psi}{\partial y^{2}} .
\end{aligned}
$$

As initial and boundaries conditions which the velocity and the temperature satisfy, we consider the following relationships

$$
\begin{aligned}
u(y, 0) & =\psi(y, 0)=0, \\
u(0, t) & =0, \\
\psi(0, t) & =t .
\end{aligned}
$$

Furthermore, we add the supplementary conditions that both the temperature $(\psi)$ and the velocity $(v)$ converge to zero when the $y$ tends to infinity. In the following Table 1, the names of the parameters used in our modeling described in this Section 3 are provided.

Table 1. Parameter descriptions

\begin{tabular}{cl}
\hline Parameters & Descriptions \\
\hline$P r$ & Prandtl number \\
\hline$G r$ & Grashof number \\
\hline$c_{p}$ & Heat at a constant pressure \\
\hline$g$ & Acceleration constant \\
\hline$\beta$ & Volumetric coefficient of thermal expansion \\
\hline$v$ & Kinematics viscosity of the fluid \\
\hline$\kappa$ & Thermal conductivity of the fluid \\
\hline$\alpha_{1}$ & Second grade parameter \\
\hline$\rho$ & Fluid density \\
\hline
\end{tabular}

\section{Analytical approaches}

In this section, we consider the initial and boundary conditions in Eqs. (22)-(24) to give the analytical solution of equations (20) and (21) via the Laplace transform method. The basic tool here is solving the second-order differential equations via the Laplace transform. The sketch of the proof consists in first getting the exact analytical solution of Eq. (21) and using this solution to determine also the analytical solution of Eq. (20).

Here we begin with Equation (21) under initial and boundary conditions described in Eqs. (22)-(24). Applying the Laplace transform to both sides of Eq. (21), we have that

$$
\begin{aligned}
s^{\alpha} \bar{\psi}-s^{\alpha-1} \psi(y, 0) & =\frac{1}{\operatorname{Pr}} \frac{\partial^{2} \bar{\phi}}{\partial y^{2}} \\
s^{\alpha} \bar{\psi} & =\frac{1}{\operatorname{Pr}} \frac{\partial^{2} \bar{\psi}}{\partial y^{2}} \\
\frac{\partial^{2} \bar{\psi}}{\partial y^{2}}-\operatorname{Prs}^{\alpha} \bar{\psi} & =0 .
\end{aligned}
$$

Before continuing the resolution we also apply the Laplace transform to the boundary condition (24), we get that $\psi(0, s)=1 / s^{2}$. Then the 
analytical solution of the second-order differential equation (21) in terms of Laplace transform is given as the following form

$$
\bar{\psi}(x, s)=\frac{\exp \left[-\chi \sqrt{\operatorname{Prs}^{\alpha}}\right]}{s^{2}} .
$$

The determination of the inverse of the Laplace transform needs to use the called wright function. This function is recalled to the preliminary section. Then the inverse of the Laplace transform given by Eq. (26) is given by

$$
\psi(x, t)=t \phi\left(2,-\alpha / 2,-\chi \sqrt{\operatorname{Pr}} t^{-\alpha / 2}\right) .
$$

We now consider a special case obtained when the order of the Caputo derivative converges to one, that is $\alpha=1$. We repeat the procedure of the solution with Eq. (25). Let consider this equation with the case $\alpha=1$, we have the following relationship

$$
\bar{\psi}(x, s)=\frac{\exp [-\chi \sqrt{\operatorname{Pr} s}]}{s^{2}} .
$$

The final step of the resolution consists to apply the inverse of the Laplace transform to both sides of Eq. (28), it yields that

$$
\psi(x, t)=\left(\frac{x^{2} \operatorname{Pr}}{2}+t\right) \operatorname{erfc}\left(\frac{x \sqrt{P r}}{2 \sqrt{t}}\right)-\frac{x \sqrt{P r t}}{2 \sqrt{\pi}} \exp \left(-\frac{x^{2} P r}{4 t}\right)
$$

The second step of the determination of the exact analytical solution of our model will finish with the resolution of Eq. (20). The method is similar to the procedure previously applied with the temperature distribution. In the step of the determination of the velocity, we apply the Laplace transform to both sides of equation (20), we get that

$$
\begin{aligned}
s^{\alpha} \bar{u}-s^{\alpha-1} \bar{u}(y, 0) & =\left(1+\beta s^{\alpha}\right) \frac{\partial^{2} \bar{u}}{\partial x^{2}}+G r \bar{\psi}, \\
s^{\alpha} \bar{u} & =\left(1+\beta s^{\alpha}\right) \frac{\partial^{2} \bar{u}}{\partial y^{2}}+G r \bar{\psi}, \\
\frac{\partial^{2} \bar{u}}{\partial y^{2}}-\frac{s^{\alpha}}{1+\beta s^{\alpha}} \bar{u} & =-\frac{G r}{1+\beta s^{\alpha}} \frac{\exp \left[-\chi \sqrt{\operatorname{Pr} s^{\alpha}}\right]}{s^{2}} .
\end{aligned}
$$

The solution in terms of the Laplace transform of the second-order differential equation (30) with initial and boundary conditions taken into account is given by the following relationship

$$
\bar{u}(x, s)=C\left[\frac{\exp \left[-\chi \sqrt{\operatorname{Prs}^{\alpha}}\right]}{s^{\alpha}}-\frac{\exp \left[-x \sqrt{\frac{s^{\alpha}}{1+\beta s^{\alpha}}}\right]}{s^{\alpha}}\right],
$$

where $C$ is given as

$$
C=-G r\left[\frac{s^{-2}}{1+\beta s^{\alpha}}-\frac{P r s^{-2}}{\beta P r s^{\alpha}+P r-1}\right] \text {. }
$$

To get the analytical solution, we have to apply the inverse of the Laplace transform to both sides of Eq.(31) and use the convolution product properties. We have the following analytical solution

$$
u(x, t)=\int_{0}^{t} a(t-\tau)(b(x, \tau)-c(x, \tau)) d \tau
$$

For obtaining the form of function, we apply the inverse of Laplace transform of the function $C$, we need to utilize the Mittag-Leffler function. That is

$$
a(x, t)=-\frac{G r t^{1+\alpha}}{\beta}\left[E_{\alpha, 2+\alpha}\left(-\frac{1}{\beta} t^{\alpha}\right)-E_{\alpha, 2+\alpha}\left(-\frac{\operatorname{Pr}-1}{\beta P r} t^{\alpha}\right)\right]
$$

We continue with the inverse of the Laplace transform of the function represented as the following form

$$
\bar{b}(x, s)=\frac{\exp \left[-\chi \sqrt{\operatorname{Pr} s^{\alpha}}\right]}{s^{\alpha}},
$$

which needs some manipulations. The inverse of the Laplace transform is given by the following relationship

$$
b(x, t)=t^{\alpha-1} \phi\left(\alpha,-\alpha / 2,-y \sqrt{\operatorname{Pr}} t^{-\alpha / 2}\right) .
$$


We finish this procedure of inverse of the Laplace transforms by inverting the function defined as the form

$$
\bar{c}(x, s)=\frac{\exp \left[-\chi \sqrt{\frac{s^{\alpha}}{1+\beta s^{\alpha}}}\right]}{s^{\alpha}}
$$

and then propose the analytical solution of the Eq. (20). The inverse of the Laplace transform to both sides of Eq. (37) is given by the following representation

$$
c(x, t)=\int_{0}^{\infty} m(y, u) \phi\left(0,-\alpha, u t^{-\alpha}\right) d u,
$$

where

$$
m(y, u)=1-\frac{2}{\pi \sqrt{\beta}} \int_{0}^{\infty} \frac{\sin (y u)}{u\left(u^{2}+1 / \sqrt{\beta}\right)} \exp \left(\frac{-u^{2} u t}{\beta\left(u^{2}+1 / \sqrt{\beta}\right)}\right) d u .
$$

We now consider a special case obtained when the order of the Caputo derivative converges to 1 that is $\alpha=1$, and the parameter $\beta=0$. Note that the parameter $\beta$ is zero when $\alpha_{1}=0$ in Eq. (14). In this case, we consider the Laplace transform described in Eq. (31) with the previous assumptions, there is

$$
\bar{u}(x, s)=\frac{G r}{\operatorname{Pr}-1}\left[\frac{\exp [-\chi \sqrt{s}]}{s^{3}}-\frac{\exp [-\chi \sqrt{\operatorname{Prs}}]}{s^{3}}\right] .
$$

We now apply the inverse of the Laplace transform, which is given by the following analytical form

$$
\begin{aligned}
u(x, t) & =\frac{G r}{\operatorname{Pr}-1} \int_{0}^{t}(t-\tau) \operatorname{erfc}\left(\frac{x}{2 \sqrt{\tau}}\right) d \tau \\
& -\frac{G r}{\operatorname{Pr}-1} \int_{0}^{t}(t-\tau) \operatorname{erfc}\left(\frac{x \sqrt{\operatorname{Pr}}}{2 \sqrt{\tau}}\right) d \tau .
\end{aligned}
$$

Before closing this section it is important to mention the method to get the Nusselt number. This number is obtained with the temperature distribution by the following formula

$$
N u=-\mathcal{L}^{-1}\left[\lim _{x \rightarrow 0} \frac{\partial \bar{\phi}(x, s)}{\partial x}\right]
$$

\section{Discussion on the findings}

In this section, we discuss the findings of the paper. We analyze the impact of the Caputo order derivative in the dynamics of the temperature and the velocity distribution. The impact of the Prandtl number, Grashof number, time, and second-grade coefficient will be discussed in terms of the variation of the Caputo derivative in detail.

\section{The temperature distribution}

We begin the discussion with the temperature distribution. In this part, the fractional-order and the Prandtl number will be analyzed in terms of their impacts on the dynamics of the temperature distribution. In this section, we consider Eq. (27) in the graphical representations. We fix the time $t=0.6$ for Figures $1 \mathrm{a}, 1 \mathrm{~b}$ and $t=10$ for Figures $2 \mathrm{a}, 2 \mathrm{~b}$, and also we consider different values of the order of the Caputo fractional operator. We have the following graphical results: We now analyze the behaviors of the dynamics presented in the previous figures. Let the time $t$ less than one, this assumption corresponds to Figures $1 \mathrm{a}, 1 \mathrm{~b}$. We observe that when the order of the Caputo derivative increases with the increase of the state $y$, we note that, the temperature distribution decreases. These dynamics can be explained by the fact for a short time the accumulation of the memory and heredity affects the diffusivity of the considered model. The increase in the order increases the diffusivity which generates a decrease in the temperature of the fluid. The second conclusion concerns that when the time is greater than 1, see Figures 2a, 2b, in this case, the accumulation of the memory effect and heredity makes the system more diffusive which affects the temperature distribution $2 \mathrm{a}, 2 \mathrm{~b}$ and causes its increase. In the considered cases in this part, we note that the Caputo derivative plays an acceleration effect in the dynamics of the temperature distribution.

Let us now analyze the impact of the Prandtl number in the dynamics of the considered fluid particularly on the temperature distribution We take two different times $t=0.6$ for Figures 3a, 3b, and $t=10$ for Figures $4 \mathrm{a}, 4 \mathrm{~b}$, different orders of the derivative have been considered and we increase the values of the Prandtl number. We have the following graphical representations: 


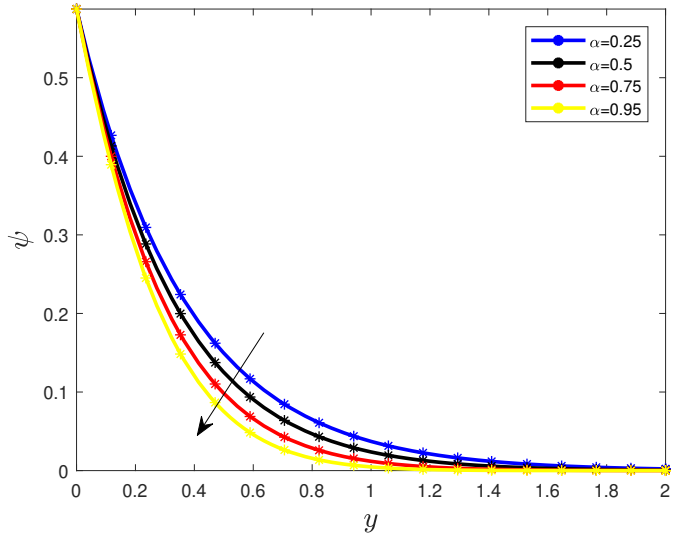

(a)

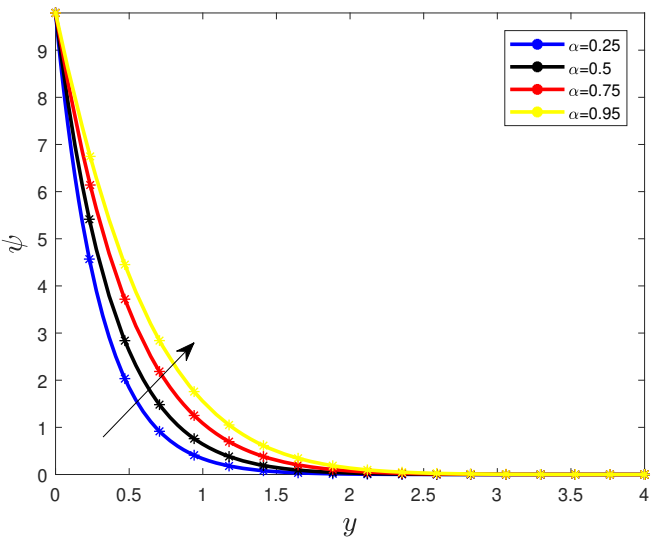

(a)

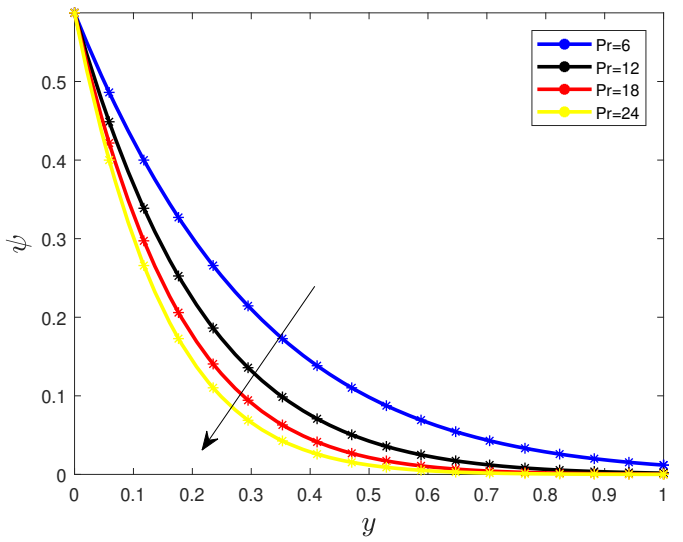

(a)

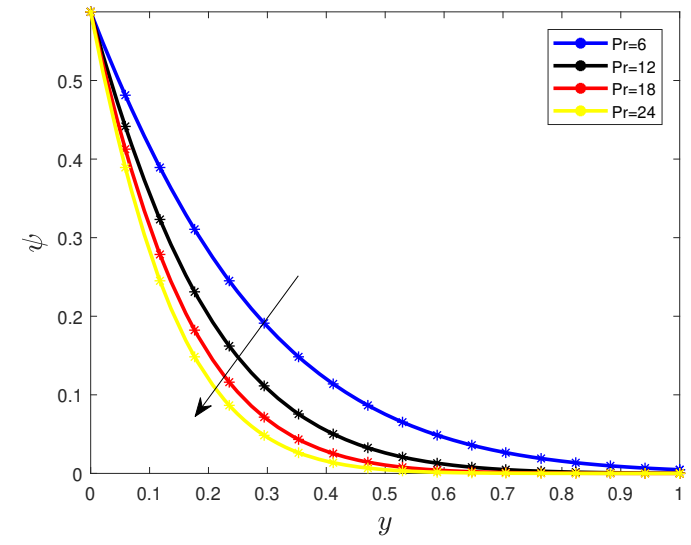

(b)

Figure 3. Temperature distribution for different values of the Prandtl number with $\alpha=0.75$ (a) and $\alpha=0.95$ (b).

The graphical representations $3 \mathrm{a}, 3 \mathrm{~b}, 4 \mathrm{a}, 4 \mathrm{~b}$ inform us that when the values of the Pr increase then temperature distribution decreases as well. These behaviors can be explained by the fact when the order of the fractional operator increases and the Prandtl number increases then the diffusivity of the system is reduced, thus its impact on the temperature distribution decreases. Let us now see what happens 


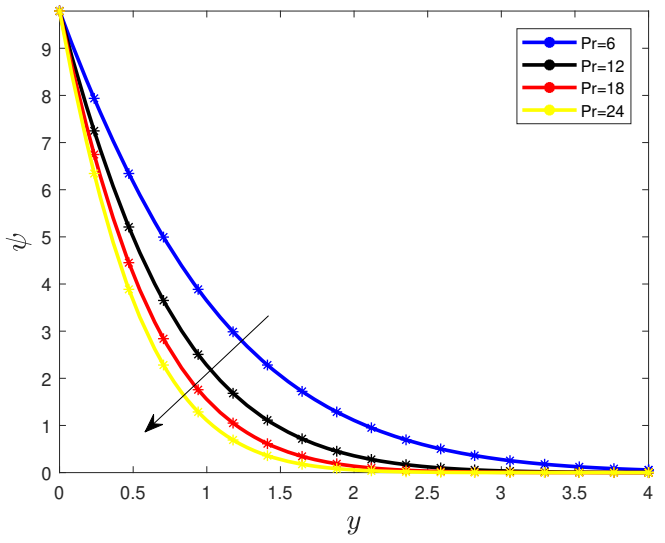

(a)

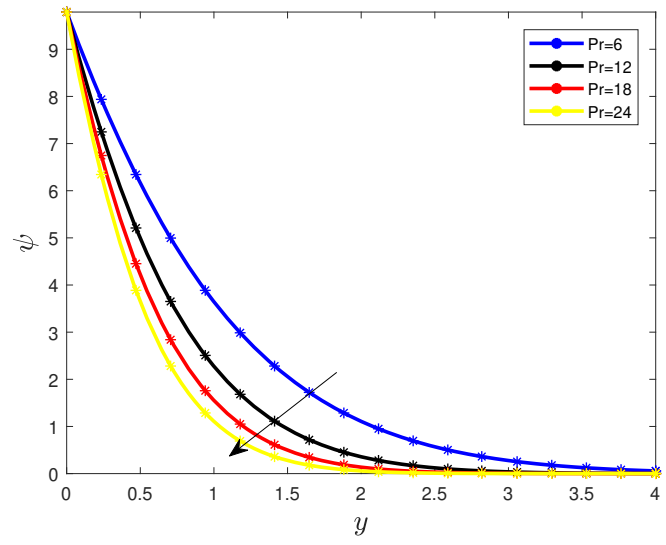

(b)

Figure 4. Temperature distribution for different values of the Prandtl number with $\alpha=0.75$ (a) and $\alpha=0.95$ (b).

with the temperature distribution when the time varies significantly and the Prandtl number increases. We have the following figures

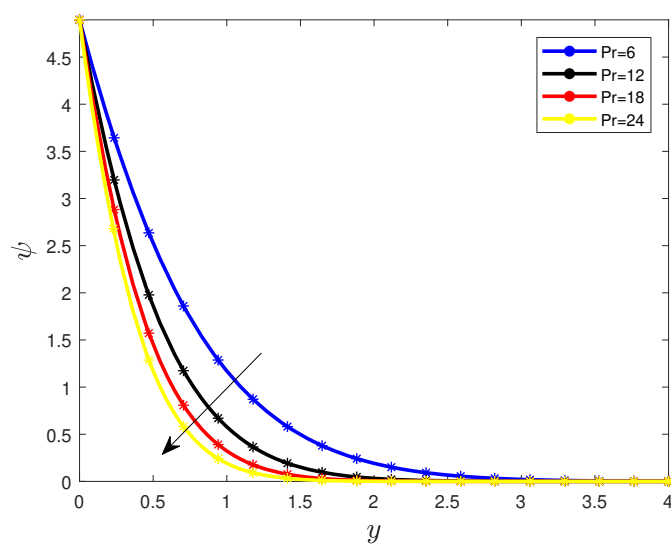

(a)

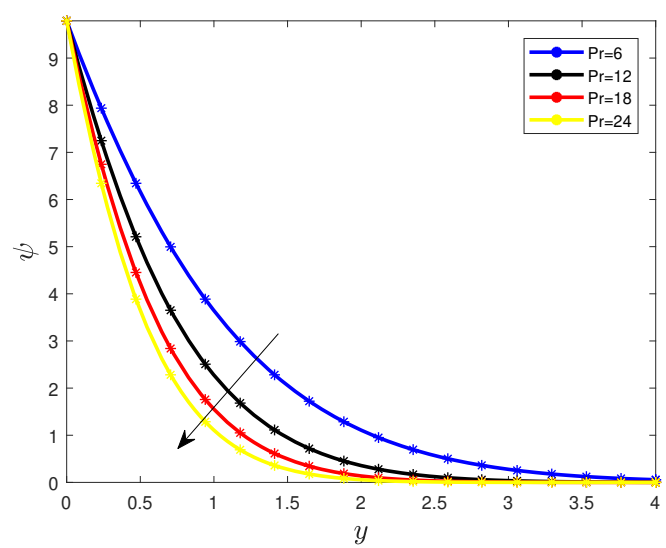

(b)

Figure 5. Temperature distribution for different values of $\operatorname{Pr}$ with $t=5$ (a) and $t=10$.

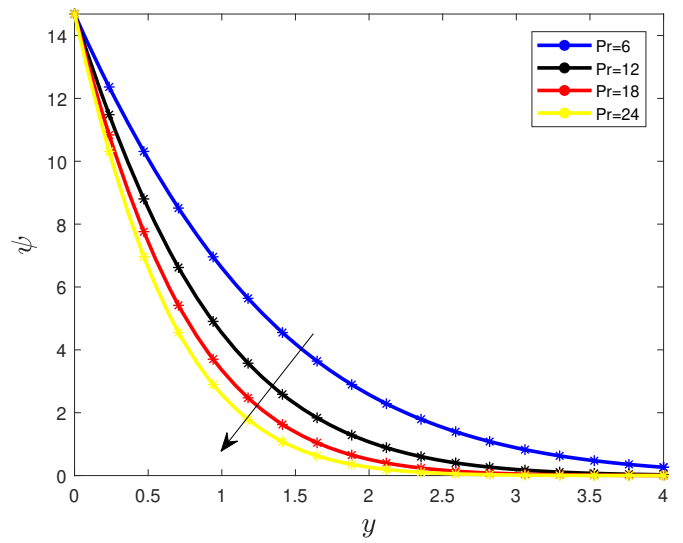

(a)

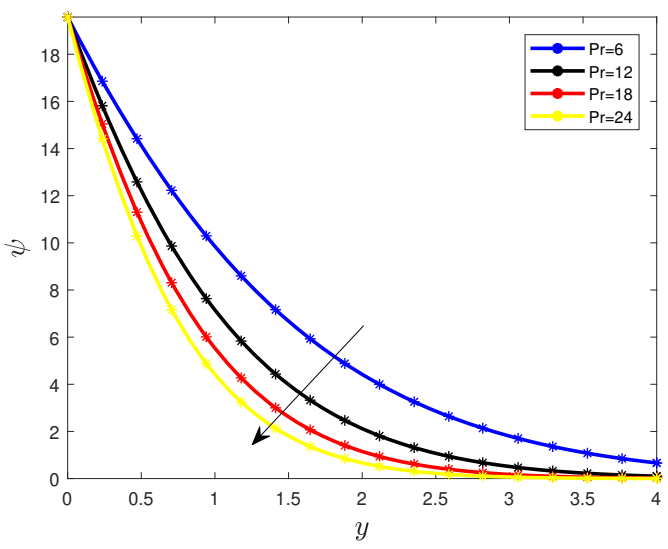

(b)

Figure 6. Temperature distribution for different values of $\operatorname{Pr}$ with $t=15$ (a) and $t=20$ (b). 
Comparing the Figures 5a, 5b, 6a, 6b, we can observe that when the Pr number increases, then the temperature distribution decreases. In conclusion, the time does not play a role if the Prandtl number increase. For all considered times the increase of the Prandtl number generates a decrease in the temperature distribution.

\section{The velocity distribution}

In this sub-section, we try to explain and interpret the dynamics generated by the velocity. We first consider analyzing the influence of the fractional-order in the dynamics. In this section, we condition Eq. (33) in the graphical representations. In the second part, we will analyze the influence of the parameters as the Grashof number $\mathrm{Gr}$ and second-grade coefficient $\beta$. Let us represent the dynamics of the velocity for different values of the Caputo fractional order in the following Figures $7 \mathrm{a}, 7 \mathrm{~b}, 8 \mathrm{a}, 8 \mathrm{~b}$. Let that $t=5$, we have the following graphics $7 \mathrm{a}, 7 \mathrm{~b}, 8 \mathrm{a}, 8 \mathrm{~b}$ for the velocity The influence of the order of the fractional derivative is analyzed in terms of the variation of the

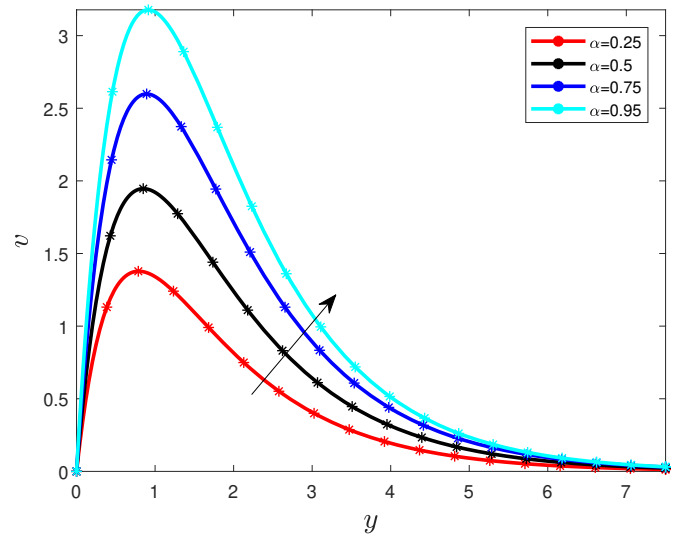

(a)

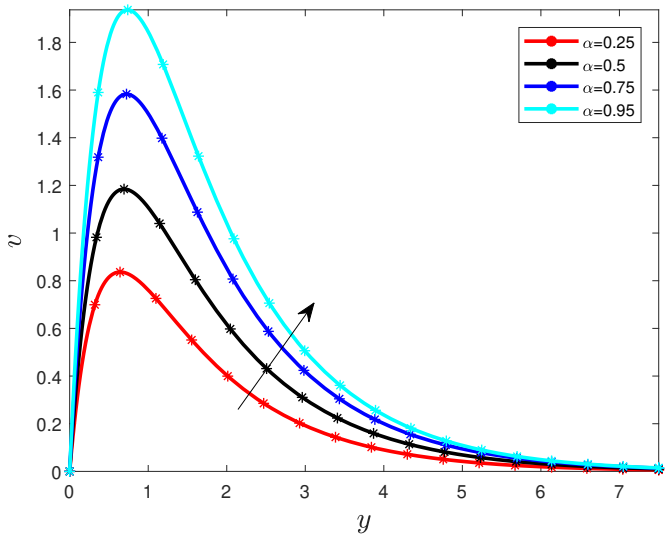

(b)

Figure 7. Velocity distribution for different values of the order $\alpha$ with $\operatorname{Pr}=5$ (a) $\operatorname{Pr}=10$ (b).

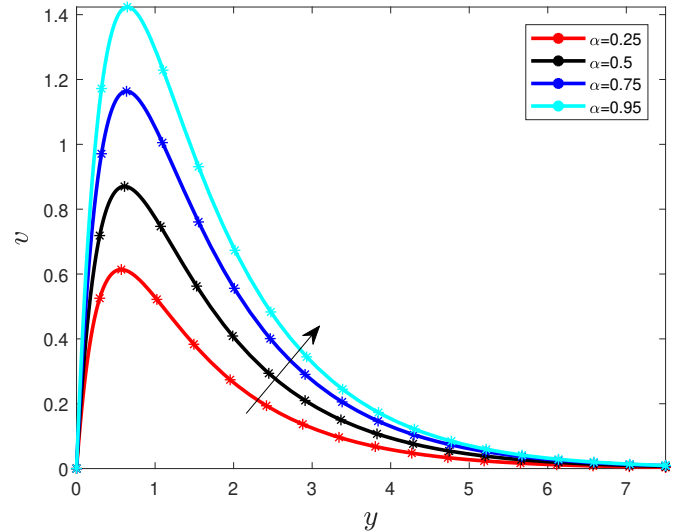

(a)

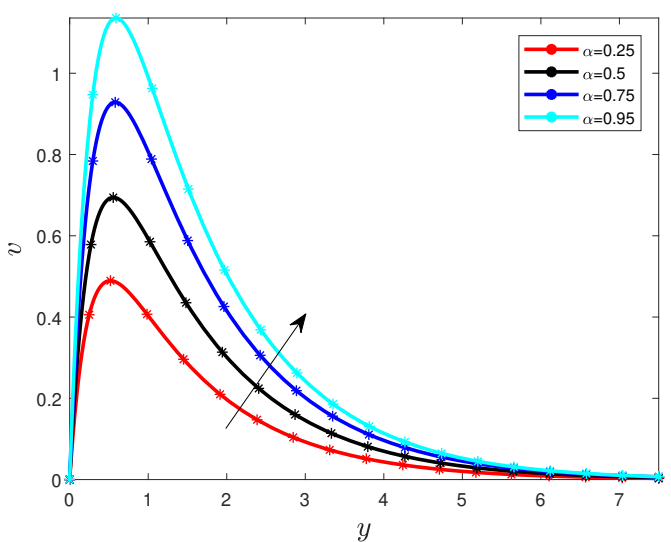

(b)

Figure 8. Velocity distribution for different values of the order $\alpha$ with $\operatorname{Pr}=15$ (a) $\operatorname{Pr}=20$ (b).

Prandtl number. We observe that when the order of the Caputo derivative increases, the velocity increases. Thus, the fractional-order has an acceleration effect in the present case. We also notice that when the Prandtl number increase, the velocity decreases, as well. The Prandtl number has the same influence on the temperature distribution and the velocity. We now consider a second case where the time is greater than $1(t=10)$ and the variation of the Grashof number is assumed. We have the following graphical representations 9a, 9b, 10a, 10b We can observe that with the previous figures the increase in the order of the Caputo derivative generates an increase in the velocity. The increase in the velocity is due to the fact when time is greater than 1, the Caputo derivative generates accumulation in the memory which causes an increase in the value of the velocity. Here, the order of the Caputo derivative has an acceleration effect. We analyze the impact of the Grashof number Gr, we can do it by analyzing the previous Figures 9a, 9b, 10a, 10b. Comparing the Figures 9a, 9b, 10a, 10b, we can observe that when the Grashof number increases, it generates an increase in velocity. This increase in the velocity is explained by the fact that when the Grashof number increases then we have increased in the thermal buoyancy force. Let us now analyze the second-grade parameter. We have 


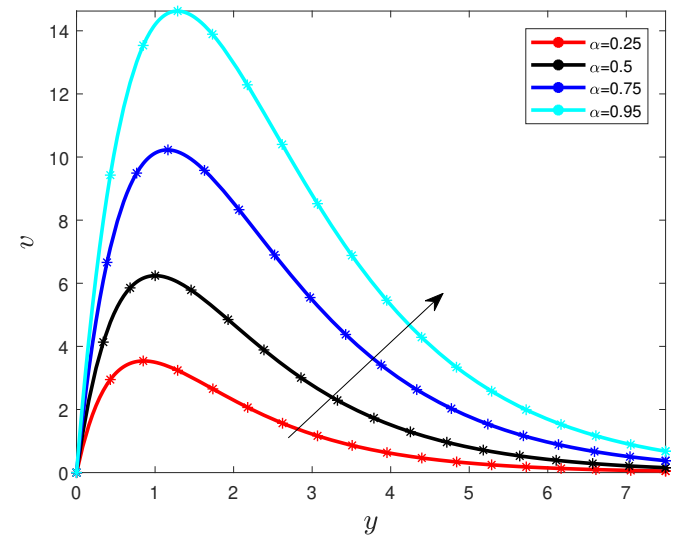

(a)

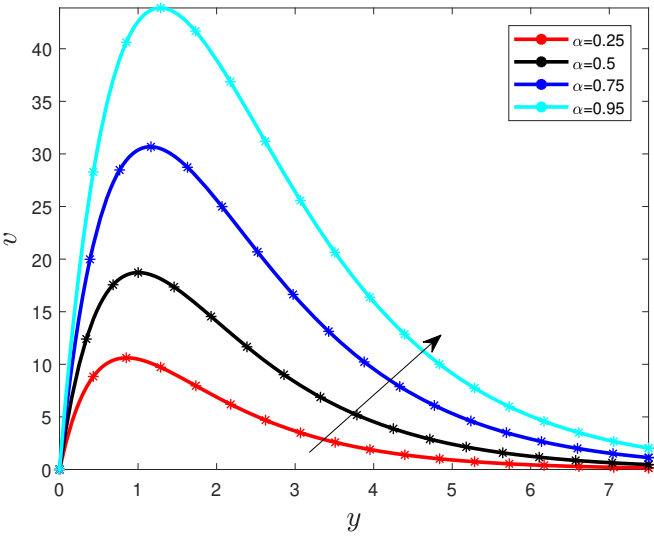

(a)

Figure 10. Velocity distribution for different values of the order $\alpha$ with $\mathrm{Gr}=15$ and $\mathrm{Gr}=20$.

Figure 9. Velocity distribution for different values of the order $\alpha$ with $\mathrm{Gr}=5$ and $\mathrm{Gr}=10$.

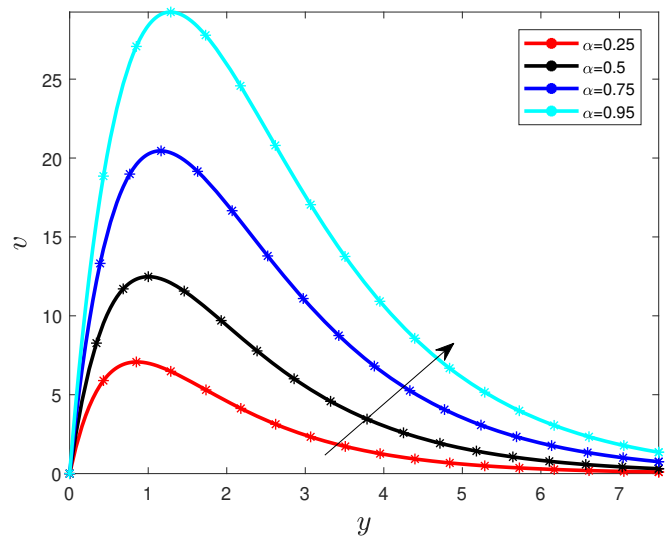

(b)

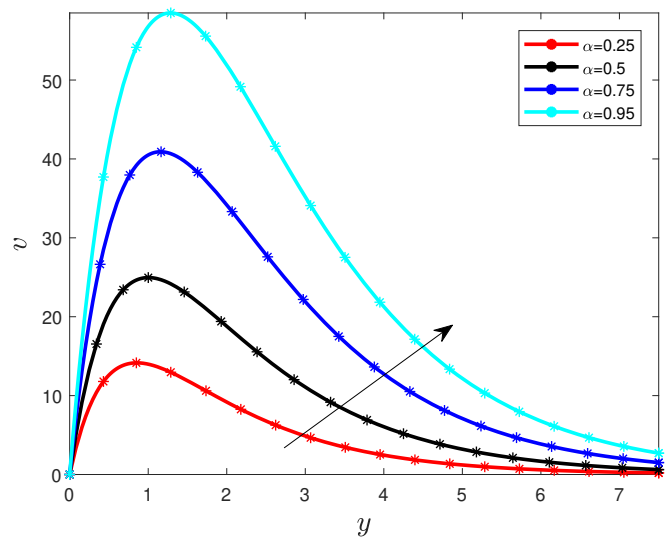

(b)

the following graphical representations $11 \mathrm{a}, 11 \mathrm{~b}, 12 \mathrm{a}, 12 \mathrm{~b}$, when the value of the second-grade parameter increases When we compare the

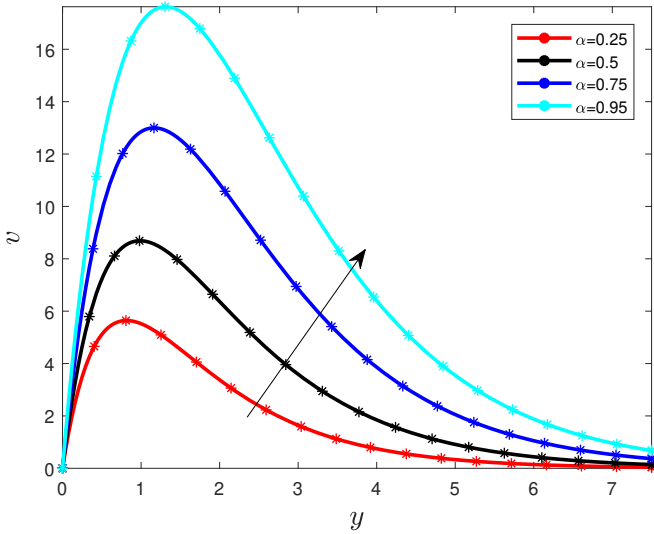

(a)

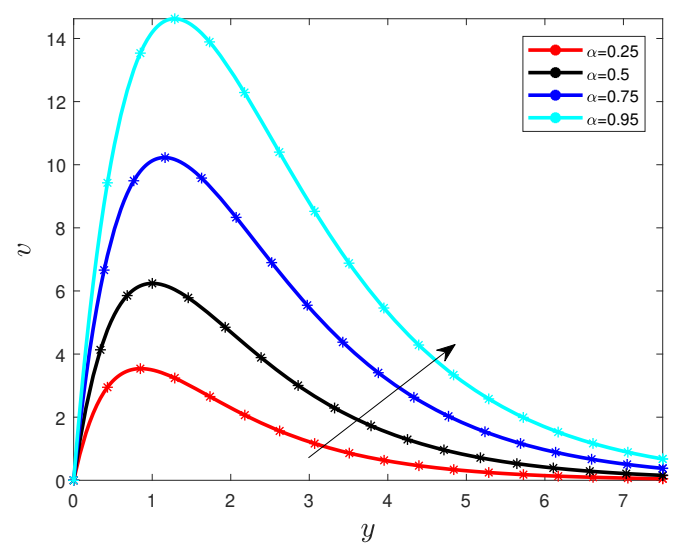

(b)

Figure 11. Velocity distribution for different values of the order $\alpha$ with $\beta=0$ (a) and $\beta=0.5$ (b).

values of the velocity in Figures 11a, 11b, 12a, 12b, we notice when the values of the second-grade parameter increase, then the velocity 


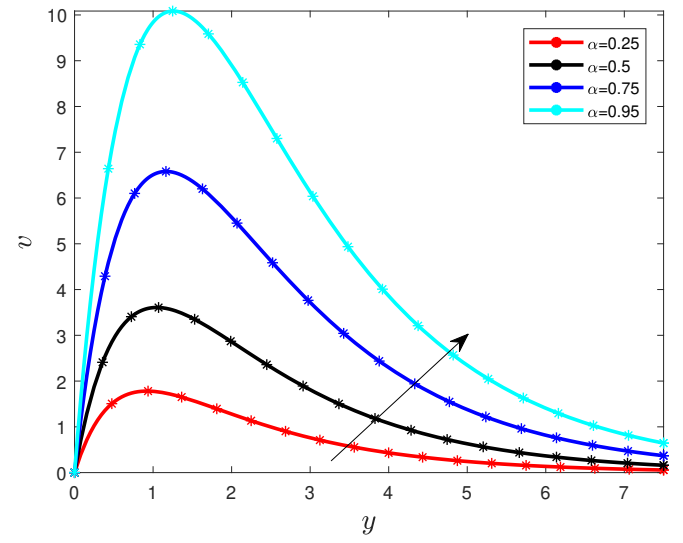

(a)

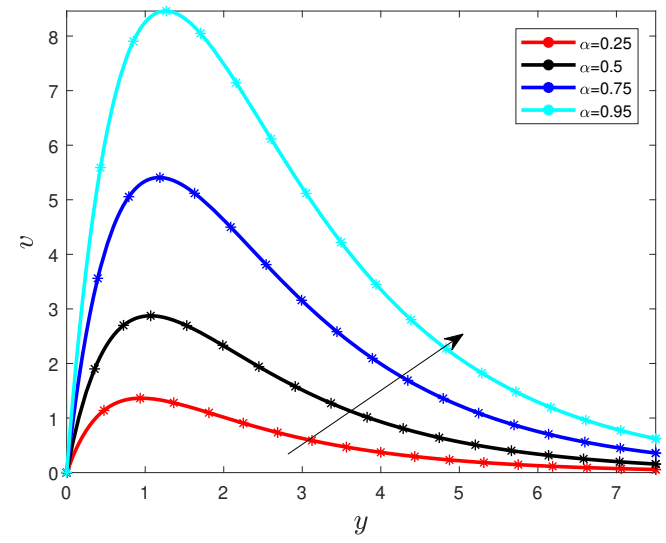

(b)

Figure 12. Velocity distribution for different values of the order $\alpha$ with $\beta=0$ (a) and $\beta=0.5$ (b).

decreases as well. This behavior is explained by the fact that in general the increase of the thickness of the boundary layer is caused by the decrease of the second-grade parameter. The present investigations are similar to the investigations provided by Shah et al in [41]. In [41], the authors consider the same model addressed in this paper with the fractional derivative with the exponential kernel. The main findings in [41] and the results in the present paper are in good agreement. One of the main advantages of the present investigations regarding the investigations existing in the literature is here we use the Caputo derivative which the application of the Laplace transform and its inverse is trivial and the expressions of the exact analytical solutions of the considered fluid model can easily be expressed via Gaussian function, exponential function, and Mittag-Leffler function.

\section{Conclusion}

In this paper, we have discussed the exact analytical solutions of the second-grade fluid model described by the Caputo fractional operator. After modeling the fluid model via Caputo derivative, we have used the Laplace transform method to get the analytical solutions of the fluid model considered in this paper. Many results have been proposed in our present paper. As the first finding, the order of the fractional operator accelerates the diffusion or can have a retardation effect, that depends on the considered time. We noticed that with the increase of the Prandtl number for a specific order of the Caputo derivative then the temperature distribution of the considered fluid decreases as well. This behavior is due to the reduction of the diffusivity as previously mentioned in the paper. Note that with the increase of Grashof number $\mathrm{Gr}$ then it generates an increase in the velocity distribution of the considered fluid. For the future direction of researches, the same second-grade fluid model can be described by non-singular fractional operators as the Atangana-Baleanu derivative and Caputo-Fabrizio derivative, and getting the exact solutions with the Laplace transform constitutes an open problem and can be focused on in the future.

\section{Declarations}

\section{Consent for publication}

Not applicable.

\section{Conflicts of interest}

The author declares that he has no known competing financial interests or personal relationships that could have appeared to influence the work reported in this paper.

\section{Funding}

The author declares that there is no funding source for the reported research.

\section{Author's contributions}

The research was carried out by the author and he accepts that the contributions and responsibilities belong to the author.

\section{Acknowledgements}

Not applicable. 


\section{References}

[1] Atangana, A., \& Araz, S.İ. Extension of Atangana-Seda numerical method to partial differential equations with integer and non-integer order. Alexandria Engineering Journal, 59(4), 2355-2370, (2020). [CrossRef]

[2] Kilbas, A.A., Srivastava, H.M., \& Trujillo, J.J. Theory and applications of fractional differential equations (Vol. 204). Elsevier, (2006).

[3] Podlubny, I. Fractional Differential Equations, Academic Press: New York, NY, USA (1999).

[4] Atangana, A. \& Baleanu, D. New fractional derivatives with nonlocal and non-singular kernel: theory and application to heat transfer model, Thermal Sciences, 20(2), 763-769, (2016). [CrossRef]

[5] Caputo, M., \& Fabrizio, M. A new definition of fractional derivative without singular kernel. Progress in Fractional Differentiation and Applications, 1(2), 1-13, (2015). [CrossRef]

[6] Saad, K.M., Baleanu, D., \& Atangana, A. New fractional derivatives applied to the Korteweg-de Vries and Korteweg-de Vries-Burger's equations. Computational and Applied Mathematics, 37(4), 5203-5216, (2018). [CrossRef]

[7] Sene, N. Theory and applications of new fractional-order chaotic system under Caputo operator. An International Journal of Optimization and Control: Theories \& Applications (IJOCTA), 12(1), 20-38, (2022). [CrossRef]

[8] Wang, X., Wang, Z., Huang, X., \& Li, Y. Dynamic analysis of a delayed fractional-order SIR model with saturated incidence and treatment functions. International Journal of Bifurcation and Chaos, 28(14), 1850180, (2018). [CrossRef]

[9] Qureshi, S., Yusuf, A., Shaikh, A.A., \& Inc, M. Transmission dynamics of varicella zoster virus modeled by classical and novel fractional operators using real statistical data. Physica A: Statistical Mechanics and its Applications, 534, 122149, (2019). [CrossRef]

[10] Sene, N. Fractional SIRI Model with Delay in Context of the Generalized Liouville-Caputo Fractional Derivative. In Mathematical Modeling and Soft Computing in Epidemiology (pp. 107-125). CRC Press, (2020).

[11] Shen, Z.H., Chu, Y.M., Khan, M.A., Muhammad, S., Al-Hartomy, O.A., \& Higazy, M. Mathematical modeling and optimal control of the COVID-19 dynamics. Results in Physics, 31, 105028, (2021). [CrossRef]

[12] Li, X.P., Wang, Y., Khan, M.A., Alshahrani, M.Y., \& Muhammad, T. A dynamical study of SARS-COV-2: A study of third wave. Results in Physics, 29, 104705, (2021). [CrossRef]

[13] Yavuz, M., \& Sene, N. Stability analysis and numerical computation of the fractional predator-prey model with the harvesting rate. Fractal and Fractional, 4(3), 35, (2020). [CrossRef]

[14] Kumar, P., \& Erturk, V.S. Dynamics of cholera disease by using two recent fractional numerical methods. Mathematical Modelling and Numerical Simulation with Applications, 1(2), 102-111, (2021). [CrossRef]

[15] Ali, F., Gohar, M., \& Khan, I. MHD flow of water-based Brinkman type nanofluid over a vertical plate embedded in a porous medium with variable surface velocity, temperature and concentration. Journal of Molecular Liquids, 223, 412-419, (2016). [CrossRef]

[16] Abro, K.A. A fractional and analytic investigation of thermo-diffusion process on free convection flow: an application to surface modification technology. The European Physical Journal Plus, 135(1), 1-14, (2020). [CrossRef]

[17] Sene, N. Stability and Convergence Analysis of Numerical Scheme for the Generalized Fractional Diffusion-Reaction Equation. In Advanced Numerical Methods for Differential Equations (pp. 1-16). CRC Press, (2021).

[18] Hammouch, Z., Yavuz, M., \& Özdemir, N. Numerical solutions and synchronization of a variable-order fractional chaotic system. Mathematical Modelling and Numerical Simulation with Applications, 1(1), 11-23, (2021). [CrossRef]

[19] Rashid, M., Kalsoom, A., Ghaffar, A., Inc, M., \& Sene, N. A Multiple Fixed Point Result for-Type Contractions in the Partially OrderedDistance Spaces with an Application. Journal of Function Spaces, 2022, 6202981, (2022). [CrossRef]

[20] Shah, N.A., Khan, I., Aleem, M., \& Imran, M.A. Influence of magnetic field on double convection problem of fractional viscous fluid over an exponentially moving vertical plate: New trends of Caputo time-fractional derivative model. Advances in Mechanical Engineering, 11(7), 1-11, (2019). [CrossRef]

[21] Sheikh, N.A., Ali, F., Saqib, M., Khan, I., Jan, S.A.A., Alshomrani, A.S., \& Alghamdi, M.S. Comparison and analysis of the Atangana-Baleanu and Caputo-Fabrizio fractional derivatives for generalized Casson fluid model with heat generation and chemical reaction. Results in physics, 7, 789-800, (2017). [CrossRef]

[22] Ahmad, H., Khan, T.A., Ahmad, I., Stanimirović, P.S., \& Chu, Y.M. A new analyzing technique for nonlinear time fractional Cauchy reaction-diffusion model equations. Results in Physics, 19, 103462, (2020). [CrossRef]

[23] Yavuz, M., \& Sene, N. Approximate solutions of the model describing fluid flow using generalized $\rho$-Laplace transform method and heat balance integral method. Axioms, 9(4), 123, (2020). [CrossRef]

[24] Li, J.F., Ahmad, I., Ahmad, H., Shah, D., Chu, Y.M., Thounthong, P., \& Ayaz, M. Numerical solution of two-term time-fractional PDE models arising in mathematical physics using local meshless method. Open Physics, 18(1), 1063-1072, (2020). [CrossRef]

[25] Naik, P.A., Eskandari, Z., \& Shahraki, H.E. Flip and generalized flip bifurcations of a two-dimensional discrete-time chemical model. Mathematical Modelling and Numerical Simulation with Applications, 1(2), 95-101, (2021). [CrossRef]

[26] Yavuz, M., \& Sene, N. Fundamental calculus of the fractional derivative defined with Rabotnov exponential kernel and application to nonlinear dispersive wave model. Journal of Ocean Engineering and Science, 6(2), 196-205, (2021). [CrossRef]

[27] Panda, S.K., Ravichandran, C., \& Hazarika, B. Results on system of Atangana-Baleanu fractional order Willis aneurysm and nonlinear singularly perturbed boundary value problems. Chaos, Solitons \& Fractals, 142, 110390, (2021). [CrossRef]

[28] Tahir, M., Imran, M.A., Raza, N., Abdullah, M., \& Aleem, M. Wall slip and non-integer order derivative effects on the heat transfer flow of Maxwell fluid over an oscillating vertical plate with new definition of fractional Caputo-Fabrizio derivatives. Results in physics, 7, 1887-1898, (2017). [CrossRef]

[29] Ahmad, I., Ahmad, H., Thounthong, P., Chu, Y.M., \& Cesarano, C. Solution of multi-term time-fractional PDE models arising in mathematical biology and physics by local meshless method. Symmetry, 12(7), 1195, (2020). [CrossRef]

[30] Li, X.P., Gul, N., Khan, M.A., Bilal, R., Ali, A., Alshahrani, M.Y., ... \& Islam, S. A new Hepatitis B model in light of asymptomatic carriers and vaccination study through Atangana-Baleanu derivative. Results in Physics, 29, 104603, (2021). [CrossRef]

[31] Nisar, K.S., Jothimani, K., Kaliraj, K., \& Ravichandran, C. An analysis of controllability results for nonlinear Hilfer neutral fractional derivatives with non-dense domain. Chaos, Solitons \& Fractals, 146, 110915, (2021). [CrossRef]

[32] Bonyah, E., Yavuz, M., Baleanu, D., \& Kumar, S. A robust study on the listeriosis disease by adopting fractal-fractional operators Alexandria Engineering Journal, 61(3), 2016-2028, (2022). [CrossRef]

[33] Vieru, D., Fetecau, C., \& Fetecau, C. Time-fractional free convection flow near a vertical plate with Newtonian heating and mass 
diffusion. Thermal Science, 19(1), 85-98, (2015). [CrossRef]

[34] Sene, N. Fractional diffusion equation with reaction term described by Caputo-Liouville generalized fractional derivative, Journal of Fractional Calculus and Applications 13(1), 42-57, (2022).

[35] Ali, F., Saqib, M., Khan, I., \& Sheikh, N.A. Application of Caputo-Fabrizio derivatives to MHD free convection flow of generalized Walters' -B fluid model. The European Physical Journal Plus, 131(10), 1-10, (2016). [CrossRef]

[36] Saqib, M., Ali, F., Khan, I., Sheikh, N.A., \& Jan, S.A.A. Exact solutions for free convection flow of generalized Jeffrey fluid: a CaputoFabrizio fractional model. Alexandria engineering journal, 57(3), 1849-1858, (2018). [CrossRef]

[37] Imran, M.A., Khan, I., Ahmad, M., Shah, N.A., \& Nazar, M. Heat and mass transport of differential type fluid with non-integer order time-fractional Caputo derivatives. Journal of Molecular Liquids, 229, 67-75, (2017). [CrossRef]

[38] Khalid, A., Khan, I., Khan, A., \& Shafie, S. Unsteady MHD free convection flow of Casson fluid past over an oscillating vertical plate embedded in a porous medium. Engineering Science and Technology, an International Journal, 18(3), 309-317, (2015). [CrossRef]

[39] Nadeem, S., Haq, R.U., \& Lee, C. MHD flow of a Casson fluid over an exponentially shrinking sheet. Scientia Iranica, 19(6), 1550-1553, (2012). [CrossRef]

[40] Narahari, M., \& Dutta, B.K. Effects of thermal radiation and mass diffusion on free convection flow near a vertical plate with Newtonian heating. Chemical Engineering Communications, 199(5), 628-643, (2012). [CrossRef]

[41] Shah, N.A., \& Khan, I. Heat transfer analysis in a second grade fluid over and oscillating vertical plate using fractional Caputo-Fabrizio derivatives. The European Physical Journal C, 76(7), 1-11, (2016). [CrossRef]

Mathematical Modelling and Numerical Simulation with Applications (MMNSA) (http://www.mmnsa.org)

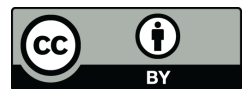

Copyright: (c) 2022 by the authors. This work is licensed under a Creative Commons Attribution 4.0 (CC BY) International License. The authors retain ownership of the copyright for their article, but they allow anyone to download, reuse, reprint, modify, distribute, and/or copy articles in MMNSA, so long as the original authors and source are credited. To see the complete license contents, please visit (http://creativecommons.org/licenses/by/4.0/). 